\title{
Constructive Theory of Scalar Characteristic Equations of the Theory of Radiation Transport: II. Algorithms for Finding Solutions and Their Analytic Representations
}

\author{
N. N. Rogovtsov \\ Belarus National Technical University, Minsk, Belarus \\ e-mail: rogovtsov@bntu.by
}

Received January 17, 2014

\begin{abstract}
We present methods for finding discrete spectra and derive analytic expressions for the eigenfunctions of scalar characteristic equations of the theory of radiation transport. We obtain new two-term recursion formulas and analytic representations for solutions of infinite tridiagonal systems of linear algebraic equations. We obtain analytic forms of the resolvents of scalar characteristic equations for phase functions square integrable on the closed interval $[-1,1]$. In addition, we derive a general analytic expression for the Green function of a two-dimensional (with respect to the angular variables) integro-differential equation of the radiation transport for the case in which the phase functions satisfy the Hölder condition on the closed interval $[-1,1]$.
\end{abstract}

DOI: $10.1134 /$ S0012266115050092

The present paper continues the paper [1] and the corresponding numbering of sections, formulas, theorems, corollaries, and remarks.

\section{ALGORITHMS FOR FINDING DISCRETE SPECTRA, EIGENFUNCTIONS, AND NORMALIZING CONSTANTS FOR REDUCED SCALAR CHARACTERISTIC EQUATIONS OF THE TRT}

The following assertion can be proved with the use of systems (4), (8), and (9), Theorems 3-6, Corollary 1 , Remark 3 , and constructions used in $[2,3]$ in the analysis and statement of properties of infinite continued fractions.

Theorem 7. Let the assumptions of Theorem 1 be true. Then the functions $\wp_{2}\left(-\zeta^{2} ; 0 ; 1\right)$ and $\wp_{0}\left(-\zeta^{2} ;|m| ; \omega_{0}\right)$, where $\left(|m|, \omega_{0}\right) \in \mathbb{Q}$, can have zeros with respect to the variable $\zeta$ only on the interval $(-1,1)$; moreover, these zeros can be only first-order zeros.

The problem of constructing well-posed efficient algorithms for separating and finding roots of any of the equations

$$
\wp_{0}\left(-\zeta^{2} ;|m| ; \omega_{0}\right)=0, \quad \wp_{2}\left(-\zeta^{2} ; 0 ; 1\right)=0
$$

[see Eqs. (6), (7), and (12)] is not necessarily simple if $\mathrm{p}(\mu) \in L_{2}(-1,1)$, because the functions $\wp_{0}\left(-\zeta^{2} ;|m| ; \omega_{0}\right)$ and $\wp_{2}\left(-\zeta^{2} ; 0 ; 1\right)$ are not polynomials. For the case in which $\left(|m| ; \omega_{0}\right) \in \mathbb{Q}$, such an algorithm was suggested and justified in [4] and was developed in [3]. It was suggested in these papers to find the roots of the above-mentioned equations on the interval $(-1,1)$ with the use of the infinite system of Sturm polynomials

$$
\left\{\ldots, \widetilde{D}_{n_{1}+1}\left(-i \zeta ;|m| ; \omega_{0}\right), \ldots, \widetilde{D}_{1}\left(-i \zeta ;|m| ; \omega_{0}\right), \widetilde{D}_{0}\left(-i \zeta ;|m| ; \omega_{0}\right)\right\}
$$


where $n_{1} \in \mathbb{N}$. For arbitrary $|m| \in \mathbb{N}_{0}$, all terms of this system were defined by the recursion formula

$$
\begin{aligned}
& \widetilde{D}_{l+1}\left(-i \zeta ;|m| ; \omega_{0}\right) \\
& =-(\ell+1)^{-1} \varkappa_{\ell}^{\times}\left(|m| ; \omega_{0}\right) \widetilde{D}_{\ell}\left(-i \zeta ;|m| ; \omega_{0}\right)-\zeta^{2}((\ell+2|m|) /(\ell+1)) \widetilde{D}_{\ell-1}\left(-i \zeta ;|m| ; \omega_{0}\right), \quad \ell \in \mathbb{N}, \\
& \widetilde{D}_{0}\left(-i \zeta ;|m| ; \omega_{0}\right)=1, \quad \widetilde{D}_{1}\left(-i \zeta ;|m| ; \omega_{0}\right)=-(2|m|+1)\left(1-\omega_{0} f_{|m|}\right) .
\end{aligned}
$$

For separating and finding the roots of the equation $\wp_{2}\left(-\zeta^{2} ; 0 ; 1\right)=0$ on the interval $(-1,1)$, one can also use the infinite system of Sturm polynomials, which, however, should be constructed with regard of the relation $\varkappa_{0}^{\times}(0 ; 1)=0$. Consider the sequence of polynomials

$$
\left\{\ldots, \widetilde{D}_{n_{1}+1}(-i \zeta ; 0 ; 1), \ldots, \widetilde{D}_{1}(-i \zeta ; 0 ; 1), \widetilde{D}_{0}(-i \zeta ; 0 ; 1)\right\}, \quad n_{1} \in \mathbb{N}
$$

where

$$
\widetilde{D}_{0}(-i \zeta ; 0 ; 1)=1, \quad \widetilde{D}_{1}(-i \zeta ; 0 ; 1)=-\varkappa_{2}^{\times}(0 ; 1)=-5\left(1-f_{2}\right) .
$$

The polynomials $\widetilde{D}_{n_{1}+1}(-i \zeta ; 0 ; 1)$ are defined by the relations

$$
\widetilde{D}_{n_{1}+1}(-i \zeta ; 0 ; 1)=\left(\left(n_{1}+1\right) !\right)^{-1} D_{n_{1}+1}(-i \zeta ; 0 ; 1),
$$

where, for any $n_{1} \in \mathbb{N}, D_{n_{1}+1}(-i \zeta ; 0 ; 1)$ is the determinant of the basic matrix of a finite system, which contains $n_{1}+1$ equations and is obtained from subsystem (9) by its truncation and the replacement of the parameter $\nu_{0}$ by the quantity $(-i \zeta)$. That truncation can be reduced to the replacement of all quantities $\left.b_{j}^{\times}\left(\nu_{0} ; 0 ; 1 ; \overrightarrow{0}_{0}\right)\right|_{j \geq n_{1}+3}$ in subsystem (9) by zeros. It follows from the definition of the polynomials $\left.\widetilde{D}_{\ell}(-i \zeta ; 0 ; 1)\right|_{\ell \in \mathbb{N}_{0}}$ that they are related to each other by the recursion formula

$$
\widetilde{D}_{n_{1}+1}(-i \zeta ; 0 ; 1)=-\frac{2 n_{1}+5}{n_{1}+1}\left(1-f_{n_{1}+2}\right) \widetilde{D}_{n_{1}}(-i \zeta ; 0 ; 1)-\frac{\left(n_{1}+2\right)^{2}}{n_{1}\left(n_{1}+1\right)} \zeta^{2} \widetilde{D}_{n_{1}-1}(-i \zeta ; 0 ; 1) \quad\left(n_{1} \in \mathbb{N}\right),
$$

where $\widetilde{D}_{0}(-i \zeta ; 0 ; 1)=1$ and $\widetilde{D}_{1}(-i \zeta ; 0 ; 1)=-5\left(1-f_{2}\right)$.

Theorem 8. Let the assumptions of Theorem 1 be satisfied, and let $m=0$ and $\omega_{0}=1$. Then the following assertions hold.

1. For each $\ell \in \mathbb{N}$, the polynomial $\widetilde{D}_{\ell+1}(-i \zeta ; 0 ; 1)$ can have only first-order zeros on the interval $(-1,1)$; moreover, they are necessarily symmetric around the point $\zeta=0$.

2. $\lim _{\ell \rightarrow+\infty} \widetilde{D}_{\ell}(-i \zeta ; 0 ; 1)=0$ if the number $\zeta$ is a zero of the function $\wp_{2}\left(-\zeta^{2} ; 0 ; 1\right)$ on $(-1,1)$.

3. The set of all zeros of the polynomial $\widetilde{D}_{n_{1}+1}(-i \zeta ; 0 ; 1)$ with any $n_{1} \in \mathbb{N}$ on the interval $(-1,1)$ coincides with the set of all zeros of the finite continued fraction

$$
\wp_{2 ; n_{1}}\left(-\zeta^{2} ; 0 ; 1\right)=\left[1 ; \frac{\left(-q_{2}(0 ; 1)\right) \zeta^{2}}{1}, \ldots, \frac{\left(-q_{n_{1}+1}(0 ; 1)\right) \zeta^{2}}{1}\right]
$$

which can have only first-order zeros on $(-1,1)$; moreover,

$$
\wp_{2}\left(-\zeta^{2} ; 0 ; 1\right)=\lim _{n_{1} \rightarrow+\infty} \wp_{2 ; n_{1}}\left(-\zeta^{2} ; 0 ; 1\right) \quad \text { for arbitrary } \zeta \in(-1,1) .
$$

4. The finite subsystem

$$
\left\{\widetilde{D}_{n_{1}+1}(-i \zeta ; 0 ; 1), \widetilde{D}_{n_{1}}(-i \zeta ; 0 ; 1), \ldots, \widetilde{D}_{0}(-i \zeta ; 0 ; 1)\right\},
$$

where $n_{1} \in \mathbb{N}$, of the infinite system of Sturm polynomials is a system of Sturm polynomials for the equation $\widetilde{D}_{n_{1}+1}(-i \zeta ; 0 ; 1)=0$ with respect to the variable $\zeta \in(0,1) ;$ moreover, the number of zeros 
of the polynomial $\widetilde{D}_{n_{1}+1}(-i \zeta ; 0 ; 1)$ on the interval $\left(\beta_{1} ; \beta_{2}\right)\left[\beta_{1}<\beta_{2} ; \beta_{1}, \beta_{2} \in(0,1)\right]$ is equal to the number of sign alternations in the finite subsystem of Sturm polynomials corresponding to $n_{1}$ when passing from $\beta_{1}$ to $\beta_{2}$.

5. If $\zeta$ is a zero of the function $\wp_{2}\left(-\zeta^{2} ; 0 ; 1\right)$ on the interval $(-1,1)$, then there exists a finite number $n_{0} \in \mathbb{N}_{0}$ such that the inequality

$$
\widetilde{D}_{n_{1}+1}(-i \zeta ; 0 ; 1) \neq 0
$$

holds for any finite number $n_{1} \geq n_{0}$.

It was shown in $[3,4]$ that if $\left(|m| ; \omega_{0}\right) \in \mathbb{Q}$, then the use of an infinite system of Sturm polynomials does not lead to the loss of a zero or to the appearance of false ones. The same result holds if $m=0$ and $\omega_{0}=1$. The validity of this assertion can be proved with the use of properties of the polynomials $\widetilde{D}_{s}\left(-i \zeta ;|m| ; \omega_{0}\right)[3,4]$ and continued fractions $[5-7]$, Theorems 2,3 , and 6 , the Perron and van Vleck theorems, the theorem on the existence of the limit of a monotone decreasing lowerbounded numerical sequence, and the method of contradiction.

Theorem 9. Suppose that the assumptions of Theorem 8 are satisfied and there exists at least one zero of the function $\wp_{2}\left(-\zeta^{2} ; 0 ; 1\right)$ on the interval $(0,1)$. Then the elements of all sequences of approximate values of zeros of the function $\wp_{2}\left(-\zeta^{2} ; 0 ; 1\right)$ that correspond to the index $\ell \in \mathbb{N}[\ell$ is the index of zero in the sequence of zeros of that function on $(0,1) ;$ moreover, they are arranged in the increasing order] and are zeros of the polynomial $\widetilde{D}_{n_{1}+1}(-i \zeta ; 0 ; 1)$ on the interval $(0,1)$ for various values of $n_{1}=1,2,3, \ldots$ are monotone decreasing with increasing $n_{1}$ and tend to the corresponding exact values of zeros of the function $\wp_{2}\left(-\zeta^{2} ; 0 ; 1\right)$ as $n_{1} \rightarrow+\infty$. In addition, on any interval $(0, \beta)$, where $\beta \in(0,1)$, for sufficiently large $n_{1}$, the use of a finite subsystem of Sturm polynomials does not lead to the loss of zero or to the appearance of false zeros for finding approximate values of zeros of the function $\wp_{2}\left(-\zeta^{2} ; 0 ; 1\right)$.

Suppose that, for some pair $\left(|m|, \omega_{0}\right) \in \mathbb{N}_{0} \times(0,1], \Re\left(|m| ; \omega_{0}\right)$ is a nonempty set and $\nu=-i \zeta \in$ $\Re\left(|m| ; \omega_{0}\right)$, where $\zeta \in(-1,1)$. Then $i \zeta \in \Re\left(|m| ; \omega_{0}\right)$. If pairs differ at least by one of values of the quantities $|m|$ and $\omega_{0}$, then their corresponding discrete spectra of scalar reduced characteristic equations do not necessarily coincide. Therefore, below elements of the set $\Re\left(|m| ; \omega_{0}\right)$ are denoted by $\zeta= \pm i \zeta\left(|m| ; \omega_{0}\right)$, where $\zeta\left(|m| ; \omega_{0}\right) \in[0,1)$, and by $\Phi_{|m|}\left( \pm i \zeta\left(|m| ; \omega_{0}\right) ; \mu ; \omega_{0}\right)$ we denote the eigenfunctions corresponding to the elements $\left( \pm i \zeta\left(|m| ; \omega_{0}\right)\right)$. Since nontrivial solutions $\Phi_{|m|}\left( \pm i \zeta\left(|m| ; \omega_{0}\right) ; \mu ; \omega_{0}\right)$ and $\left\{b_{s}^{\times}\left( \pm i \zeta\left(|m| ; \omega_{0}\right) ;|m| ; \omega_{0} ; \overrightarrow{0}_{|m|}\right)\right\}$ of the corresponding homogeneous analogs in the families of the IE (3) and SLAE (4) in the classes $L_{2}(-1,1)$ and $\ell_{2 ; K_{2}(|m|)}$ are defined to within an arbitrary nonzero factor, we introduce the coordinated normalizations

$$
\begin{gathered}
b_{0}^{\times}\left( \pm i \zeta\left(|m| ; \omega_{0}\right) ;|m| ; \omega_{0} ; \overrightarrow{0}_{|m|}\right)=[(2|m|) !]^{1 / 2} \\
\int_{-1}^{1}\left(1-\mu^{2}\right)^{|m| / 2} \Phi_{|m|}\left( \pm i \zeta\left(|m| ; \omega_{0}\right) ; \mu ; \omega_{0}\right) d \mu=2^{|m|}(|m|) ![(2|m|) !]^{-1} .
\end{gathered}
$$

Theorem 10. Let the assumptions of Theorem 1 be satisfied, and let $\Re\left(|m| ; \omega_{0}\right) \neq \varnothing$. Then the eigenfunctions $\Phi_{|m|}\left( \pm i \zeta\left(|m| ; \omega_{0}\right) ; \mu ; \omega_{0}\right)$ of the corresponding homogeneous analog of the IE in the family of IE (3) under the normalization condition (18) belong to the class $C[-1,1]$ and can be represented in the analytic form

$$
\begin{aligned}
\Phi_{|m|}( & \left. \pm i \zeta\left(|m| ; \omega_{0}\right) ; \mu ; \omega_{0}\right) \\
& =\left[2((2|m|) !)^{1 / 2}\right]^{-1} \sum_{s=0}^{+\infty}(2(s+|m|)+1) \Psi_{s}^{\times}\left( \pm i \zeta\left(|m| ; \omega_{0}\right) ;|m| ; \omega_{0}\right) \mathrm{P}_{s+|m|,|m|}^{\times}(\mu) \\
& =\omega_{0}\left[2((2|m|) !)^{1 / 2}\left(1 \pm \zeta\left(|m| ; \omega_{0}\right) \mu\right)\right]^{-1}
\end{aligned}
$$




$$
\begin{gathered}
\times \sum_{s=0}^{+\infty}(2(s+|m|)+1) f_{s+|m|} \Psi_{s}^{\times}\left( \pm i \zeta\left(|m| ; \omega_{0}\right) ;|m| ; \omega_{0}\right) \mathrm{P}_{s+|m|,|m|}^{\times}(\mu) \\
\left(|m| ; \omega_{0}\right) \in \mathbb{Q} \\
\left.\Phi_{0}( \pm i \zeta(0 ; 1) ; \mu ; 1)\right|_{\zeta(0 ; 1) \neq 0}=\frac{1}{2}\left[1-\frac{1}{2} \sum_{s=0}^{+\infty}(2 s+5) \Psi_{s}^{\wedge}( \pm i \zeta(0 ; 1) ; 0 ; 1) \mathrm{P}_{s+2}(\mu)\right] \\
=[2(1 \pm \zeta(0 ; 1) \mu)]^{-1}\left[1-\frac{1}{2} \sum_{s=0}^{+\infty}(2 s+5) f_{s+2} \Psi_{s}^{\wedge}( \pm i \zeta(0 ; 1) ; 0 ; 1) \mathrm{P}_{s+2}(\mu)\right] \\
\Phi_{0}(0 ; \mu ; 1)=2^{-1}
\end{gathered}
$$

In addition, the series in (19) and (20) are convergent pointwise and uniformly on the interval $[-1,1]$, and the quantities $\Psi_{s}^{\times}\left(\nu ;|m| ; \omega_{0}\right)$ and $\Psi_{s}^{\wedge}(\nu ; 0 ; 1)$ are defined by the relations

$$
\begin{aligned}
& \left.\Psi_{0}^{\times}\left(\nu ;|m| ; \omega_{0}\right)\right|_{|m| \in \mathbb{N}_{0}}=1,\left.\quad \Psi_{s}^{\times}\left(\nu ;|m| ; \omega_{0}\right)\right|_{s \in \mathbb{N},|m| \in \mathbb{N}_{0}} \\
& =(i \nu)^{s} \prod_{\ell=1}^{s} \varepsilon_{\ell}^{\times}(|m|)\left(\varkappa_{\ell}^{\times}\left(|m| ; \omega_{0}\right) \wp_{\ell}\left(\nu^{2} ;|m| ; \omega_{0}\right)\right)^{-1}, \\
& \Psi_{0}^{\wedge}(\nu ; 0 ; 1)=1,\left.\quad \Psi_{s}^{\wedge}(\nu ; 0 ; 1)\right|_{s \in \mathbb{N}}=(i \nu)^{s} \prod_{\ell=1}^{s}(\ell+2)\left(\varkappa_{\ell+2}^{\times}(0 ; 1) \wp_{\ell+2}\left(\nu^{2} ; 0 ; 1\right)\right)^{-1} .
\end{aligned}
$$

For the derivation of asymptotic solutions of BVP for a scalar RTE with the use of the Case method [8] or the GIRRM [9,10], one needs the normalization constants

$$
c\left( \pm i \zeta\left(|m| ; \omega_{0}\right) ;|m| ; \omega_{0}\right)=\left[\int_{-1}^{1} \mu\left(\Phi_{|m|}\left( \pm i \zeta\left(|m| ; \omega_{0}\right) ; \mu ; \omega_{0}\right)\right)^{2} d \mu\right]^{-1}
$$

Closed-form analytic expressions for these constants were obtained in $[2,3]$ for the case in which $\left(|m|, \omega_{0}\right) \in \mathbb{Q}$. By using formulas (19) and (20) and the orthogonality of the systems of associated Legendre functions, we show that the following relations hold under the assumptions of Theorem 10 :

$$
\begin{aligned}
& \left.\left(c\left( \pm i \zeta\left(|m| ; \omega_{0}\right) ;|m| ; \omega_{0}\right)\right)^{-1}\right|_{\left(|m|, \omega_{0}\right) \in \mathbb{Q}} \\
& =\mp\left(2 \zeta\left(|m| ; \omega_{0}\right)(2|m|) !\right)^{-1} \sum_{s=0}^{+\infty}(2(s+|m|)+1)\left(1-\omega_{0} f_{s+|m|}\right)\left(\Psi_{s}^{\times}\left( \pm i \zeta\left(|m| ; \omega_{0}\right) ;|m| ; \omega_{0}\right)\right)^{2} \\
& \left.(c( \pm i \zeta(0 ; 1) ; 0 ; 1))^{-1}\right|_{\zeta(0,1) \neq 0}=\mp(8 \zeta(0,1))^{-1} \sum_{s=0}^{+\infty}(2 s+5)\left(1-f_{s+2}\right)\left(\Psi_{s}^{\wedge}( \pm i \zeta(0,1) ; 0 ; 1)\right)^{2},
\end{aligned}
$$

where $\Psi_{s}^{\times}\left(\nu ;|m| ; \omega_{0}\right)$ and $\Psi_{s}^{\wedge}(\nu ; 0 ; 1)$ should be found with the use of formulas (21) and (22).

\section{ANALYTIC REPRESENTATIONS AND RECURSION FORMULAS FOR SOLUTIONS OF INFINITE SLAE}

Finite SLAE obtained with the use of the truncation from the infinite SLAE (4), (8)-(9), and (14) do not necessarily satisfy, for $\nu \in \Upsilon$, sufficient conditions of the well-posedness and stability $[11$, p. 78$]$, whose validity would provide the efficiency of the right Thomas method [11, p. 74]. An analytic method for solving inhomogeneous SLAE related bijectively with system (4) was suggested in $[2]$ for the case in which $\left(|m|, \omega_{0}\right) \in \mathbb{Q}$. This method was developed in [3], where its well-posedness and efficiency were proved for finding solutions of such infinite systems. In particular, it was shown in [3] that the solutions of these systems and the values of infinite continued fractions are stable under sufficiently small changes in the norms of phase functions in the classes 
$L_{2}(-1,1)$ and $C[-1,1]$. Note that, for applications of the TRT, it is especially interesting to develop analytic methods for solving infinite SLAE of the type (4), which would permit one to construct convenient numerical real-time algorithms for "practically arbitrary" phase functions. The use of general ideas and constructions of the GIRRM $[9,10]$ and the method suggested in $[2,3]$ permits one to develop a new well-posed effective analytic method for infinite SLAE of the type (4) and (14).

Theorem 11. Let the assumptions of Theorem 6 be true, let $\vec{v}(\nu) \in \mathrm{W}_{\ell_{2 ; \mathrm{K}}}(G)$, and let $\vec{b}(\nu ; \vec{v}(\nu))=\left\{b_{s}(\nu ; \vec{v}(\nu))\right\}_{s \in \mathbb{N}_{0}}$ be the unique solution of system (14) in $\mathrm{W}_{\ell_{2 ; \mathrm{K}}}(G)$. Then the following recursion formulas and analytic representations hold for each $\nu \in G$ :

$$
\begin{aligned}
\left.b_{s}(\nu ; \vec{v}(\nu))\right|_{s \in \mathbb{N}} & =u_{s}(\nu) b_{s-1}(\nu ; \vec{v}(\nu))+\sigma_{s}(\nu), \quad u_{s}(\nu)=i \nu \xi_{s}\left(\varkappa_{s} \wp_{s}\left(\nu^{2}\right)\right)^{-1}, \\
b_{0}(\nu ; \vec{v}(\nu)) & =\left(\varkappa_{0} \wp_{0}\left(\nu^{2}\right)\right)^{-1}\left[\psi_{0} v_{0}(\nu)+\sum_{j=1}^{+\infty}(i \nu)^{j} \psi_{j} v_{j}(\nu)\left[\prod_{\ell=1}^{j} \varepsilon_{\ell}\left(\varkappa_{\ell} \wp_{\ell}\left(\nu^{2}\right)\right)^{-1}\right]\right] \\
\left.\sigma_{s}(\nu)\right|_{s \in \mathbb{N}} & =\left(\varkappa_{s} \wp_{s}\left(\nu^{2}\right)\right)^{-1}\left[\psi_{s} v_{s}(\nu)+\sum_{j=1}^{+\infty}(i \nu)^{j} \psi_{j+s} v_{j+s}(\nu)\left[\prod_{\ell=1}^{j} \varepsilon_{\ell+s}\left(\varkappa_{\ell+s} \wp_{\ell+s}\left(\nu^{2}\right)\right)^{-1}\right]\right], \\
\left.b_{s}(\nu ; \vec{v}(\nu))\right|_{s \in \mathbb{N}} & =\left(\prod_{\ell=1}^{s} u_{\ell}(\nu)\right) b_{0}(\nu ; \vec{v}(\nu))+\sum_{j=1}^{s} \chi_{s ; j}(\nu) \sigma_{j}(\nu) \\
\left.\sigma_{s+1}(\nu)\right|_{s \in \mathbb{N}} & =\left(i \nu \varepsilon_{s+1}\right)^{-1}\left[\varkappa_{s} \wp_{s}\left(\nu^{2}\right) \sigma_{s}(\nu)-\psi_{s} v_{s}(\nu)\right] .
\end{aligned}
$$

In addition, $\sigma_{1}(\nu)$ is defined by the last formula in $(23)$, and the $\chi_{s ; j}(\nu)$ are defined by the relations $\left.\chi_{s ; s}(\nu)\right|_{s \in \mathbb{N}}=1$ and $\chi_{s ; j}(\nu)=\prod_{\ell=j+1}^{s} u_{\ell}(\nu)$ for arbitrary $s \in \mathbb{N} \backslash\{1\}$ and arbitrary $j \in\{1, \ldots, s-1\}$.

Proof. By virtue of assumption 5 of Theorem 6 , for each $\vec{v}(\nu) \in \mathrm{W}_{\ell_{2 ; K}}(G)$, there exists a unique solution $\vec{b}(\nu ; \vec{v}(\nu))$ of system (14) in $\mathrm{W}_{\ell_{2 ; \mathrm{K}}}(G)$. Therefore, $\vec{b}(\nu ; \vec{v}(\nu)) \in \ell_{2 ; \mathrm{K}}$ for each $\nu \in G$. It follows from the proof of Theorem 6 that for each $\nu \in G$ the component $b_{0}(\nu ; \vec{v}(\nu))$ of this solution is an analytic function and is defined by formula (17), which is similar to the second formula in (23).

Let us show that the first and third formulas in (23) hold. Since, for any $\nu \in G$, the quantity $b_{0}(\nu ; \vec{v}(\nu))$ is defined by the second formula in (23), we find that it can be considered to be known. In the second equation of system (14), we transpose the term $i \nu \xi_{1} b_{0}(\nu ; \vec{v}(\nu))$ to the right-hand side and rewrite this system without the first equation in the form

$$
\begin{aligned}
i \nu \varepsilon_{1}^{(1)} b_{1}^{(1)}(\nu ; \vec{v}(\nu)) & =\varkappa_{0}^{(1)} b_{0}^{(1)}(\nu ; \vec{v}(\nu))-\tau_{0}^{(1)}(\nu), \\
i \nu \varepsilon_{2}^{(1)} b_{2}^{(1)}(\nu ; \vec{v}(\nu))+i \nu \xi_{1}^{(1)} b_{0}^{(1)}(\nu ; \vec{v}(\nu)) & =\varkappa_{1}^{(1)} b_{1}^{(1)}(\nu ; \vec{v}(\nu))-\tau_{1}^{(1)}(\nu), \\
i \nu \varepsilon_{3}^{(1)} b_{3}^{(1)}(\nu ; \vec{v}(\nu))+i \nu \xi_{2}^{(1)} b_{1}^{(1)}(\nu ; \vec{v}(\nu)) & =\varkappa_{2}^{(1)} b_{2}^{(1)}(\nu ; \vec{v}(\nu))-\tau_{2}^{(1)}(\nu), \ldots
\end{aligned}
$$

Here

$$
\begin{aligned}
b_{s}^{(1)}(\nu ; \vec{v}(\nu)) & =b_{s+1}(\nu ; \vec{v}(\nu)) \quad \text { for any } \quad s \in \mathbb{N}_{0} ; \\
\tau_{s}^{(1)}(\nu) & =\tau_{s+1}(\nu) \quad \text { for any } \quad s \in \mathbb{N} \text { and } \tau_{0}^{(1)}(\nu)=i \nu \xi_{1} b_{0}(\nu ; \vec{v}(\nu))+\tau_{1}(\nu) ; \\
\varepsilon_{s-1}^{(1)} & =\varepsilon_{s} \text { and } \xi_{s-1}^{(1)}=\xi_{s} \quad \text { for any } \quad s \in \mathbb{N} \backslash\{1\} ; \quad \varkappa_{s-1}^{(1)}=\varkappa_{s} \text { for any } s \in \mathbb{N} .
\end{aligned}
$$

System (26) has the same form as system (14). By virtue of the existence of a unique solution of system (14) in $\ell_{2 ; \mathrm{K}}$ for any $\nu \in G$ and the definition of the class $\ell_{2 ; \mathrm{K}}$, system (26) has a unique solution $\left.\left\{b_{s}^{(1)}(\nu ; \vec{v}(\nu))\right\}\right|_{s \in \mathbb{N}_{0}}$ in $\ell_{2 ; K}$ for any $\nu \in G$ as well. For any $\nu \in G$, this solution coincides with the subsequence $\vec{b}^{(1)}(\nu ; \vec{v}(\nu))=\left.\left\{b_{s}(\nu ; \vec{v}(\nu))\right\}\right|_{s \in \mathbb{N}}$ of the unique solution $\left.\left\{b_{s}(\nu ; \vec{v}(\nu))\right\}\right|_{s \in \mathbb{N}_{0}}$ of system (14) in $\ell_{2 ; \mathrm{K}}$. This, together with the relation $\left.\left\{b_{s}(\nu ; \vec{v}(\nu))\right\}\right|_{s \in \mathbb{N}_{0}} \in \mathrm{W}_{\ell_{2 ; K}}(G)$, implies the existence and uniqueness of the solution of system (26) in $\mathrm{W}_{\ell_{2 ; \mathrm{K}}}(G)$. Therefore, all assumptions 
of Theorem 6 are satisfied for system (26). By Theorem 6 and the above-introduced notation, formula (17) acquires the form

$$
b_{1}(\nu ; \vec{v}(\nu))=b_{0}^{(1)}(\nu ; \vec{v}(\nu))=\left(\varkappa_{0}^{(1)} \wp_{0}^{(1)}\left(\nu^{2}\right)\right)^{-1} \sum_{j=0}^{+\infty} \Xi_{j}^{(1)}(\nu) \tau_{j}^{(1)}(\nu)
$$

here

$$
\begin{aligned}
& \wp_{\ell}^{(1)}\left(\nu^{2}\right)=\left[1 ; \frac{q_{\ell}^{(1)} \nu^{2}}{1}, \frac{q_{\ell+1}^{(1)} \nu^{2}}{1}, \ldots\right], \quad \ell \in \mathbb{N}_{0}, \quad q_{\ell}^{(1)}=\varepsilon_{\ell+1}^{(1)} \xi_{\ell+1}^{(1)}\left(\varkappa_{\ell}^{(1)} \varkappa_{\ell+1}^{(1)}\right)^{-1}, \\
& \Psi_{0}^{(1)}(\nu)=1,\left.\quad \Psi_{\ell}^{(1)}(\nu)\right|_{\ell \in \mathbb{N}}=(i \nu)^{\ell} \prod_{r=1}^{\ell} \xi_{r}^{(1)}\left(\varkappa_{r}^{(1)} \wp_{r}^{(1)}\left(\nu^{2}\right)\right)^{-1} ; \\
& \Xi_{0}^{(1)}(\nu)=1,\left.\quad \Xi_{j}^{(1)}(\nu)\right|_{j \in \mathbb{N}}=\left[\prod_{r=1}^{j} \frac{\varepsilon_{r}^{(1)}}{\xi_{r}^{(1)}}\right] \Psi_{j}^{(1)}(\nu) .
\end{aligned}
$$

Let us reproduce all above-described manipulations for system (26) under the assumption that $b_{0}^{(1)}(\nu ; \vec{v}(\nu))$ is known. As a result, we obtain a system of the same form as (26). By reproducing such manipulations for the transformed systems with regard of formula (17), the definition of the class $\ell_{2 ; K}$, the existence and uniqueness of the solution of any transformed system for any $\vec{v}(\nu) \in \mathrm{W}_{\ell_{2 ; K}}(G)$ in $\mathrm{W}_{\ell_{2 ; K}}(G)$, by taking into account the invariance of their forms (under the above-described actions), and by using induction, we obtain

$$
b_{s}(\nu ; \vec{v}(\nu))=b_{0}^{(s)}(\nu ; \vec{v}(\nu))=\left(\varkappa_{0}^{(s)} \wp_{0}^{(s)}\left(\nu^{2}\right)\right)^{-1} \sum_{j=0}^{+\infty} \Xi_{j}^{(s)}(\nu) \tau_{j}^{(s)}(\nu), \quad(s, \nu) \in \mathbb{N}_{0} \times G \text {. }
$$

Here $b_{0}^{(0)}(\nu ; \vec{v}(\nu))=b_{0}(\nu ; \vec{v}(\nu)) ; \wp_{0}^{(s)}\left(\nu^{2}\right)=\wp_{s}\left(\nu^{2}\right), s \in \mathbb{N}_{0} ; \Xi_{0}^{(s)}(\nu)=1, s \in \mathbb{N}_{0}$, and $\Xi_{j}^{(s)}(\nu)=$ $(i \nu)^{j} \prod_{\ell=1}^{j} \varepsilon_{\ell+s}\left(\varkappa_{\ell+s} \wp_{\ell+s}\left(\nu^{2}\right)\right)^{-1},(s, j) \in \mathbb{N}_{0} \times \mathbb{N} ; \tau_{0}^{(s)}(\nu)=i \nu \xi_{s} b_{s-1}(\nu ; \vec{v}(\nu))+\psi_{s} v_{s}(\nu), s \in \mathbb{N}$, $\tau_{0}^{(0)}=\tau_{0}(\nu)=\psi_{0} v_{0}(\nu)$ and $\tau_{j}^{(s)}(\nu)=\tau_{j+s}(\nu)=\psi_{j+s} v_{j+s}(\nu),(s, j) \in \mathbb{N} \times \mathbb{N}$. However, to within notation, the first and third formulas in (23) are identical to formula (27) for all $s \in \mathbb{N}$. Thus, all formulas in (23) are true. Formulas (23) readily imply formulas (24). The recursion formula (25) can be proved by a straightforward comparison of the expressions for $\sigma_{s}(\nu)$ for $s=n$ and $s=n+1$ $(n \in \mathbb{N})$ and by using induction. The proof of the theorem is complete. hold.

Corollary 2. Let the assumptions of Theorem 11 be satisfied, and let the following conditions

1. The limits $\lim _{s \rightarrow+\infty}\left(\varepsilon_{s+1} / \varkappa_{s}\right)$ and $\lim _{s \rightarrow+\infty}\left(\xi_{s} / \varkappa_{s}\right)$ are finite and nonzero.

2. There exists an $s_{0} \in \mathbb{N}$ such that the inequality

$$
\left|\varkappa_{s}\right| \prod_{\ell=1}^{s}\left|\varepsilon_{\ell} / \xi_{\ell}\right| \geq \text { const }>0
$$

holds for arbitrary $s \geq s_{0}$.

3. For each $\nu \in G$, the characteristic equation corresponding to system (14) without the first equation has roots with distinct absolute values.

Then, for each $\nu \in G$, the sequence $\left\{\sigma_{s}(\nu)\right\}_{s \in \mathbb{N}_{0}}$, where $\sigma_{0}(\nu)$ is an arbitrary finite number and $\sigma_{s}(\nu)$ is defined by the third formula in $(23)$ for any $s \in \mathbb{N}$, belongs to the class $\ell_{2 ; \mathrm{K}}$, and there exists an $s_{1} \in \mathbb{N}$ such that the inequality $\left|u_{s}(\nu)\right| \leq$ const $<1$ holds for each $s \geq s_{1}$.

Corollary 3. Suppose that assumptions of Corollary 2 are satisfied and in addition, for some $\nu \in G$ and for a finite $n \in \mathbb{N}_{0}$, the sequence $\vec{b}\left(\nu ; \vec{v}_{[0 ; n] ; \infty}(\nu)\right)$ is the unique solution of system (14) 
in $\ell_{2 ; \mathrm{K}}$ for $\vec{v}(\nu)=\vec{v}_{[0 ; n] ; \infty}(\nu)$. Then, for a given $\nu \in G$, this solution can be constructed with the use of the following algorithm.

1. If $n=0$, then

$b_{0}\left(\nu ; \vec{v}_{[0 ; 0] ; \infty}(\nu)\right)=\left(\varkappa_{0} \wp_{0}\left(\nu^{2}\right)\right)^{-1} \psi_{0} v_{0}(\nu),\left.\quad b_{s}\left(\nu ; \vec{v}_{[0 ; 0] ; \infty}(\nu)\right)\right|_{s \in \mathbb{N}}=\left(\prod_{\ell=1}^{s} u_{\ell}(\nu)\right) b_{0}\left(\nu ; \vec{v}_{[0 ; 0] ; \infty}(\nu)\right)$.

2. If $n=1$, then

$$
\begin{aligned}
b_{0}\left(\nu ; \vec{v}_{[0 ; 1] ; \infty}(\nu)\right) & =\left(\varkappa_{0} \wp_{0}\left(\nu^{2}\right)\right)^{-1}\left(\psi_{0} v_{0}(\nu)+\psi_{1} v_{1}(\nu) \Xi_{1}(\nu)\right), \quad \sigma_{1}(\nu)=\left(\varkappa_{1} \wp_{1}\left(\nu^{2}\right)\right)^{-1} \psi_{1} v_{1}(\nu), \\
\left.b_{s}\left(\nu ; \vec{v}_{[0 ; 1] ; \infty}(\nu)\right)\right|_{s \in \mathbb{N}} & =u_{s}(\nu) b_{s-1}\left(\nu ; \vec{v}_{[0 ; 1] ; \infty}(\nu)\right)+\delta_{1 s} \sigma_{1}(\nu), \\
\left.b_{s}\left(\nu ; \vec{v}_{[0 ; 1] ; \infty}(\nu)\right)\right|_{s \in \mathbb{N}} & =\left(\prod_{\ell=1}^{s} u_{\ell}(\nu)\right) b_{0}\left(\nu ; \vec{v}_{[0 ; 1] ; \infty}(\nu)\right)+\chi_{s ; 1}(\nu) \sigma_{1}(\nu) .
\end{aligned}
$$

3. If $n \geq 2$, then

$$
\begin{aligned}
b_{0}\left(\nu ; \vec{v}_{[0 ; n] ; \infty}(\nu)\right) & =\left(\varkappa_{0} \wp_{0}\left(\nu^{2}\right)\right)^{-1} \sum_{\ell=0}^{n} \Xi_{\ell}(\nu) \psi_{\ell} v_{\ell}(\nu), \\
\left.\sigma_{s}(\nu)\right|_{s \in\{n, \ldots, 1\}} & =\left(\varkappa_{s} \wp_{s}\left(\nu^{2}\right)\right)^{-1}\left[i \nu \varepsilon_{s+1} \sigma_{s+1}(\nu)+\psi_{s} v_{s}(\nu)\right],\left.\quad \sigma_{s}(\nu)\right|_{s \geq n+1}=0, \\
\left.b_{s}\left(\nu ; \vec{v}_{[0 ; n] ; \infty}(\nu)\right)\right|_{s \in \mathbb{N}} & =u_{s}(\nu) b_{s-1}\left(\nu ; \vec{v}_{[0 ; n] ; \infty}(\nu)\right)+\sigma_{s}(\nu), \\
\left.b_{s}\left(\nu ; \vec{v}_{[0 ; n] ; \infty}(\nu)\right)\right|_{s \in \mathbb{N}} & =\left(\prod_{\ell=1}^{s} u_{\ell}(\nu)\right) b_{0}\left(\nu ; \vec{v}_{[0 ; n] ; \infty}(\nu)\right)+\sum_{j=1}^{\theta(s ; n)} \chi_{s ; j}(\nu) \sigma_{j}(\nu),
\end{aligned}
$$

where $\theta(s ; n)=s$ for $s \in\{1, \ldots, n\}$ and $\theta(s ; n)=n$ for $s>n$.

Remark 4. Suppose that the assumptions of Corollary 2 are satisfied, for some $\nu \in G$, the sequence $\left\{v_{s}(\nu)\right\}_{s \in \mathbb{N}_{0}}$ contains a countable subset of nonzero elements, and $\left\{v_{j_{r}}(\nu)\right\}_{r \in \mathbb{N}}$ is a subsequence of all such elements; moreover, $j_{r} \in \mathbb{N}_{0}$ and $j_{r_{1}}<j_{r_{2}}$ for arbitrary $r_{1}<r_{2}$. Then the relation

$$
b_{s}(\nu ; \vec{v}(\nu))=\lim _{r \rightarrow+\infty} b_{s}\left(\nu ; \vec{v}_{\left[0 ; j_{r}\right] ; \infty}(\nu)\right)
$$

holds for any finite number $s \in \mathbb{N}_{0}$, where $\vec{v}_{\left[0 ; j_{r} ; \infty\right.}(\nu)=\left(v_{0}(\nu), \ldots, v_{j_{r}}(\nu), 0,0,0, \ldots\right)$. In addition, for any finite $r \in \mathbb{N}$, the quantity $b_{s}\left(\nu ; \vec{v}_{\left[0 ; j_{r}\right] ; \infty}(\nu)\right)$ can be found with the use of the algorithm described in Corollary 3.

The above assertions permit one to find analytic representations of solutions of the inhomogeneous systems (4) for arbitrary $\left(m, \omega_{0}, \nu\right) \in \mathbb{Z} \times(0,1] \times\left(\Upsilon \backslash \Re\left(|m| ; \omega_{0}\right)\right)$ if $\mathrm{p}(\mu) \in L_{2}(-1,1)$ and $\vec{g}_{m}^{\times} \in \ell_{2 ; K}$ for any $m \in \mathbb{Z}$. By taking into account the above-stipulated assumptions, the validity of the relations $\left(|m|, \omega_{0}\right) \in \mathbb{Q}$, Theorems $1-5$, and Remark 3, one can readily prove the validity of all assumptions of Theorem 6 and Corollary 2. Therefore, for the solution of system (4), one can use Theorem 11, Corollary 3, and Remark 4. We have thereby proved the following assertion.

Theorem 12. Let the following conditions be satisfied.

1. $\left(|m|, \omega_{0}\right) \in \mathbb{Q}$ and $\nu \in G\left(|m| ; \omega_{0}\right)=\Upsilon \backslash \Re\left(|m| ; \omega_{0}\right)$.

2. The nonnegative function $\mathrm{p}(\mu)$ belongs to $L_{2}(-1,1)$, and $\int_{-1}^{1} \mathrm{p}(\mu) d \mu=2$.

3. $g_{s}^{\times}\left(\nu ; m ; \omega_{0}\right)=g_{s}^{\vee}\left(|m| ; \mu_{1}\right)=f_{s+|m|} \mathbf{P}_{s+|m|,|m|}^{\times}\left(\mu_{1}\right)$ for arbitrary $(s,|m|) \in \mathbb{N}_{0} \times \mathbb{N}_{0}$, where $\mu_{1} \in[-1,1]$.

4. The sequence

$$
\vec{g}_{|m|}^{\vee}=\left\{f_{s+|m|} \mathrm{P}_{s+|m|,|m|}^{\times}\left(\mu_{1}\right)\right\}_{s \in \mathbb{N}_{0}}
$$

contains finitely many nonzero elements, and $n^{*}$ is the maximum nonnegative integer such that $f_{n^{*}} \mathrm{P}_{n^{*},|m|}^{\times}\left(\mu_{1}\right) \neq 0\left(n^{*} \geq|m|\right)$. 
5. The sequence

$$
\vec{b}\left(\nu ; m ; \omega_{0} ; \vec{g}_{m}^{\times}\right)=\left\{b_{s}^{\times}\left(\nu ; m ; \omega_{0} ; \vec{g}_{|m|}^{\times}\right)\right\}_{s \in \mathbb{N}_{0}}=\left\{b_{s}^{\times}\left(\nu ;|m| ; \mu_{1} ; \omega_{0} ;\left.\vec{g}_{|m|}^{\vee}\right|_{\left[0 ; n^{*}-|m|\right] ; \infty}\right)\right\}
$$

is the unique solution of system (4) in $\mathrm{W}_{\ell_{2 ; \mathrm{K}_{2}(|m|)}}\left(G\left(|m| ; \omega_{0}\right)\right)$ for

$$
\vec{g}_{m}^{\times}=\vec{g}_{|m|_{\left.\left|0 ; n^{*}-\right| m \mid\right] ; \infty}^{\vee}}^{\vee}=\left\{g_{0}^{\vee}\left(|m| ; \mu_{1}\right), \ldots, g_{n^{*}-|m|}^{\vee}\left(|m| ; \mu_{1}\right), 0,0,0, \ldots\right\}
$$

Then the desired solution of system (4) in $\mathrm{W}_{\ell_{2 ; \mathrm{K}_{2}(|m|)}}\left(G\left(|m| ; \omega_{0}\right)\right)$ can be found with the use of the following analytic representations and recursion formulas.

1. If $n^{*}=|m|$, then

$$
\begin{aligned}
b_{0}^{\times}\left(\nu ;|m| ; \mu_{1} ; \omega_{0} ; \vec{g}_{|m|_{[0 ; 0] ; \infty}^{\vee}}^{\vee}\right. & =u_{0}^{\times}\left(\nu ;|m| ; \omega_{0}\right) \varkappa_{0}^{\times}(|m| ; 0) g_{0}^{\vee}\left(|m| ; \mu_{1}\right), \\
\left.b_{s}^{\times}\left(\nu ;|m| ; \mu_{1} ; \omega_{0} ; \vec{g}_{|m|_{[0 ; 0] ; \infty}^{\vee}}^{\vee}\right)\right|_{s \in \mathbb{N}} & =\left(\prod_{l=1}^{s} u_{l}^{\times}\left(\nu ;|m| ; \omega_{0}\right)\right) b_{0}^{\times}\left(\nu ;|m| ; \mu_{1} ; \omega_{0} ; \vec{g}_{|m|_{[0 ; 0] ; \infty}}\right),
\end{aligned}
$$

where

$$
\begin{aligned}
u_{0}^{\times}\left(\nu ;|m| ; \omega_{0}\right) & =\left(\varkappa_{0}^{\times}\left(|m| ; \omega_{0}\right) \wp_{0}\left(\nu^{2} ;|m| ; \omega_{0}\right)\right)^{-1}, \\
\left.u_{s}^{\times}\left(\nu ;|m| ; \omega_{0}\right)\right|_{s \in \mathbb{N}} & =i \nu \varepsilon_{s}^{\times}(|m|)\left(\varkappa_{s}^{\times}\left(|m| ; \omega_{0}\right) \wp_{s}\left(\nu^{2} ;|m| ; \omega_{0}\right)\right)^{-1} .
\end{aligned}
$$

2. If $n^{*}=|m|+1$, then

$$
\begin{aligned}
& b_{0}^{\times}\left(\nu ;|m| ; \mu_{1} ; \omega_{0} ; \vec{g}_{|m|[0 ; 1] ; \infty}^{\vee}\right) \\
& =u_{0}^{\times}\left(\nu ;|m| ; \omega_{0}\right)\left[\varkappa_{0}^{\times}(|m| ; 0) g_{0}^{\vee}\left(|m| ; \mu_{1}\right)+u_{1}^{\times}\left(\nu ;|m| ; \omega_{0}\right) \varkappa_{1}^{\times}(|m| ; 0) g_{1}^{\vee}\left(|m| ; \mu_{1}\right)\right] \text {, } \\
& \sigma_{1}^{\times}\left(\nu ;|m| ; \mu_{1} ; \omega_{0}\right)=\left(i \nu \varepsilon_{1}^{\times}(|m|)\right)^{-1} u_{1}^{\times}\left(\nu ;|m| ; \omega_{0}\right) \varkappa_{1}^{\times}(|m| ; 0) g_{1}^{\vee}\left(|m| ; \mu_{1}\right), \\
& \left.b_{s}^{\times}\left(\nu ;|m| ; \mu_{1} ; \omega_{0} ; \vec{g}_{|m|[0 ; 1] ; \infty}^{\vee}\right)\right|_{s \in \mathbb{N}} \\
& =u_{s}^{\times}\left(\nu ;|m| ; \omega_{0}\right) b_{s-1}^{\times}\left(\nu ;|m| ; \mu_{1} ; \omega_{0} ;\left.\vec{g}_{|m|}^{\vee}\right|_{\mid 0 ; 1 ; \infty \infty}\right)+\delta_{1 s} \sigma_{1}^{\times}\left(\nu ;|m| ; \mu_{1} ; \omega_{0}\right), \\
& \left.b_{s}^{\times}\left(\nu ;|m| ; \mu_{1} ; \omega_{0} ; \vec{g}_{|m|_{[0 ; 1] ; \infty}^{\vee}}^{\vee}\right)\right|_{s \in \mathbb{N}} \\
& =\left(\prod_{\ell=1}^{s} u_{\ell}^{\times}\left(\nu ;|m| ; \omega_{0}\right)\right) b_{0}^{\times}\left(\nu ;|m| ; \mu_{1} ; \omega_{0} ; \vec{g}_{|m|_{[0 ; 1] ; \infty}}\right)+\chi_{s ; 1}^{\times}\left(\nu ;|m| ; \omega_{0}\right) \sigma_{1}^{\times}\left(\nu ;|m| ; \mu_{1} ; \omega_{0}\right),
\end{aligned}
$$

where the quantity $\chi_{s ; j}^{\times}\left(\nu ;|m| ; \omega_{0}\right)$ is defined by the relations

$$
\left.\chi_{s ; s}^{\times}(\nu)\right|_{s \in \mathbb{N}}=1, \quad \chi_{s ; j}^{\times}\left(\nu ;|m| ; \omega_{0}\right)=\prod_{\ell=j+1}^{s} u_{\ell}^{\times}\left(\nu ;|m| ; \omega_{0}\right)
$$

for arbitrary $s \in \mathbb{N} \backslash\{1\}$ and for arbitrary $j \in\{1, \ldots, s-1\}$.

3. If $n^{*} \geq|m|+2$, then

$$
\begin{aligned}
& b_{0}^{\times}\left(\nu ;|m| ; \mu_{1} ; \omega_{0} ; \vec{g}_{\left.|m|_{\left.\left|0 ; n^{*}-\right| m \mid\right] ; \infty}^{\vee}\right)}^{\vee}=\sum_{\ell=0}^{n^{*}-|m|}\left[\prod_{r=0}^{\ell} u_{r}^{\times}\left(\nu ;|m| ; \omega_{0}\right)\right] \varkappa_{\ell}^{\times}(|m| ; 0) \vec{g}_{\ell}^{\vee}\left(|m| ; \mu_{1}\right),\right. \\
& \left.\sigma_{s}^{\times}\left(\nu ;|m| ; \mu_{1} ; \omega_{0}\right)\right|_{s \in\left\{n^{*}-|m|, \ldots, 1\right\}} \\
& =\left(i \nu \varepsilon_{s}^{\times}(|m|)\right)^{-1} u_{s}^{\times}\left(\nu ;|m| ; \omega_{0}\right)\left[i \nu \varepsilon_{s+1}^{\times}(|m|) \sigma_{s+1}^{\times}\left(\nu ;|m| ; \mu_{1} ; \omega_{0}\right)+\varkappa_{s}^{\times}(|m| ; 0) g_{s}^{\vee}\left(|m| ; \mu_{1}\right)\right] \text {, } \\
& \left.\sigma_{s}^{\times}\left(\nu ;|m| ; \mu_{1} ; \omega_{0}\right)\right|_{s \geq n^{*}-|m|+1}=0, \\
& \left.b_{s}^{\times}\left(\nu ;|m| ; \mu_{1} ; \omega_{0} ; \vec{g}_{|m|_{\left[0 ; n^{*}-|m|\right] ; \infty}^{\vee}}^{\vee}\right)\right|_{s \in \mathbb{N}} \\
& =u_{s}^{\times}\left(\nu ;|m| ; \omega_{0}\right) b_{s-1}^{\times}\left(\nu ;|m| ; \mu_{1} ; \omega_{0} ; \vec{g}_{|m|_{\left[0 ; n^{*}-|m|\right] ; \infty}^{\vee}}^{\vee}\right)+\sigma_{s}^{\times}\left(\nu ;|m| ; \mu_{1} ; \omega_{0}\right),
\end{aligned}
$$




$$
\begin{aligned}
b_{s}^{\times}\left(\nu ;|m| ; \mu_{1} ; \omega_{0} ; \vec{g}_{\left.|m|_{\left.\left|0 ; n^{*}-\right| m \mid\right] ; \infty}^{\vee}\right)\left.\right|_{s \in \mathbb{N}}}\right. \\
=\left(\prod_{\ell=1}^{s} u_{\ell}^{\times}\left(\nu ;|m| ; \omega_{0}\right)\right) b_{0}^{\times}\left(\nu ;|m| ; \mu_{1} ; \omega_{0} ; \vec{g}_{\left.|m|_{\left[0 ; n^{*}-|m|\right] ; \infty}^{\vee}\right)}\right) \\
\quad+\sum_{j=1}^{\theta\left(s ; n^{*}-|m|\right)} \chi_{s ; j}^{\times}\left(\nu ;|m| ; \omega_{0}\right) \sigma_{j}^{\times}\left(\nu ;|m| ; \mu_{1} ; \omega_{0}\right) .
\end{aligned}
$$

Remark 5. Let assumptions 1-3 of Theorem 12 be satisfied, and let

$$
\vec{b}^{\times}\left(\nu ; m ; \omega_{0} ; \vec{g}_{m}^{\times}\right)=\vec{b}^{\times}\left(\nu ;|m| ; \mu_{1} ; \omega_{0} ; \vec{g}_{|m|}^{\vee}\right)=\left\{\vec{b}_{s}^{\times}\left(\nu ;|m| ; \mu_{1} ; \omega_{0} ; \vec{g}_{|m|}^{\vee}\right)\right\}_{s \in \mathbb{N}_{0}}
$$

be the unique solution of system (4) in $W_{\ell_{2 ; K_{2}|| m|\rangle}}\left(G\left(|m| ; \omega_{0}\right)\right)$ for the case in which the sequence

$$
\vec{g}_{m}^{\times}=\vec{g}_{|m|}^{\vee}=\left\{f_{s+|m|} \mathrm{P}_{s+|m| ;|m|}^{\times}\left(\mu_{1}\right)\right\}_{s \in \mathbb{N}_{0}}
$$

contains a countable subset of nonzero elements and $\left\{f_{j_{r}+|m|} \mathrm{P}_{j_{r}+|m| ;|m|}^{\times}\left(\mu_{1}\right)\right\}_{r \in \mathbb{N}}$ is a subsequence of all such elements; moreover, $j_{r} \in \mathbb{N}_{0}$ and $j_{r_{1}}<j_{r_{2}}$ for arbitrary $r_{1}<r_{2}$. Then the relation

$$
b_{s}^{\times}\left(\nu ;|m| ; \mu_{1} ; \omega_{0} ; \vec{g}_{|m|}^{\vee}\right)=\lim _{r \rightarrow+\infty} b_{s}^{\times}\left(\nu ;|m| ; \mu_{1} ; \omega_{0} ; \vec{g}_{|m|_{\left[0 ; j_{r}\right] ; \infty}^{\vee}}^{\vee}\right)
$$

holds for any finite $s \in \mathbb{N}_{0}$, where

$$
\vec{g}_{|m|_{\left|0 ; j_{r}\right| ; \infty}}=\left(f_{|m|} \mathrm{P}_{|m|,|m|}^{\times}\left(\mu_{1}\right), \ldots, f_{j_{r}+|m|} \mathrm{P}_{j_{r}+|m|,|m|}^{\times}\left(\mu_{1}\right), 0,0,0, \ldots\right) .
$$

In this case, for any finite $r \in \mathbb{N}$ and for $\nu \in G\left(|m| ; \omega_{0}\right)$, the quantity $b_{s}^{\times}\left(\nu ;|m| ; \mu_{1} ; \omega_{0} ; \vec{g}_{\left.|m|_{\left[0 ; j_{r}\right] ; \infty}\right)}\right.$ can be found with the use of the algorithm described in Theorem 12 .

If $m=0$ and $\omega_{0}=1$, then $\varkappa_{0}^{\times}(0 ; 1)=0$. Then system (4) does not satisfy assumption 1 of Theorem 6. In this case, system (4) splits in the finite subsystem consisting of its first two equations and the infinite subsystem containing all remaining equations. That infinite subsystem, in view of the equations of the finite subsystem, can be reduced to an infinite system that has the same form as system (4). If, in addition, $\nu \in G(0 ; 1)=\Upsilon \backslash \Re(0 ; 1)$ and $\left.g_{s}^{\times}(\nu ; 0 ; 1)\right|_{s \in \mathbb{N}_{0}}=\left.g_{s}^{\vee}\left(0 ; \mu_{1}\right)\right|_{s \in \mathbb{N}_{0}}=f_{s} \mathrm{P}_{s}\left(\mu_{1}\right)$, then such an infinite system satisfies all assumptions of Theorem 6 and Corollary 2 . We have thereby proved the following assertion.

Theorem 13. Let $m=0, \omega_{0}=1$, and $\nu \in G(0 ; 1)$, and let assumptions $2-5$ of Theorem 12 be satisfied. Then the unique solution of system (4) in $\mathrm{W}_{\ell_{2 ; K_{2}(0)}}(G(0 ; 1))$ can be found with the use of the following analytic representations and recursion formulas.

1. The relations

$$
\begin{aligned}
& b_{0}^{\times}\left(\nu ; 0 ; \mu_{1} ; 1 ; \vec{g}_{0}^{\vee}\right)=-(i \nu)^{-1}\left[3\left(1-f_{1}\right)(i \nu)^{-1}+3 f_{1} \mathrm{P}_{1}\left(\mu_{1}\right)+2 i \nu b_{2}^{\times}\left(\nu ; 0 ; \mu_{1} ; 1 ; \vec{g}_{0}^{\vee}\right)\right], \\
& b_{1}^{\times}\left(\nu ; 0 ; \mu_{1} ; 1 ; \vec{g}_{0}^{\vee}\right)=-(i \nu)^{-1}
\end{aligned}
$$

hold for arbitrary $n^{*} \in \mathbb{N}_{0} ;$ moreover, $f_{1} \mathrm{P}_{1}\left(\mu_{1}\right)=0$ for $n^{*}=0$.

2. If $n^{*} \in\{0 ; 1 ; 2\}$, then

$$
\begin{aligned}
b_{2}^{\times}\left(\nu ; 0 ; \mu_{1} ; 1 ; \vec{g}_{0}^{\vee}\right) & =\left(\varkappa_{2}^{\times}(0 ; 1) \wp_{2}\left(\nu^{2} ; 0 ; 1\right)\right)^{-1}\left[-2+5 f_{2} \mathrm{P}_{2}\left(\mu_{1}\right) \delta_{n^{*} 2}\right], \\
\left.b_{s+2}^{\times}\left(\nu ; 0 ; \mu_{1} ; 1 ; \vec{g}_{0}^{\vee}\right)\right|_{s \in \mathbb{N}} & =\left[\prod_{\ell=3}^{s+2} u_{\ell}^{\times}(\nu ; 0 ; 1)\right] b_{2}^{\times}\left(\nu ; 0 ; \mu_{1} ; 1 ; \vec{g}_{0}^{\vee}\right) .
\end{aligned}
$$

3. If $n^{*}=3$, then

$$
\begin{aligned}
b_{2}^{\times}\left(\nu ; 0 ; \mu_{1} ; 1 ; \vec{g}_{0}^{\vee}\right) & =\left(\varkappa_{2}^{\times}(0 ; 1) \wp_{2}\left(\nu^{2} ; 0 ; 1\right)\right)^{-1}\left[-2+5 f_{2} \mathrm{P}_{2}\left(\mu_{1}\right)+7 f_{3} \mathrm{P}_{3}\left(\mu_{1}\right) u_{3}^{\times}(\nu ; 0 ; 1)\right], \\
\left.b_{s+2}^{\times}\left(\nu ; 0 ; \mu_{1} ; 1 ; \vec{g}_{0}^{\vee}\right)\right|_{s \in \mathbb{N}} & =u_{s+2}^{\times}(\nu ; 0 ; 1) b_{s+1}^{\times}\left(\nu ; 0 ; \mu_{1} ; 1 ; \vec{g}_{0}^{\vee}\right)+\delta_{s 1} U\left(\nu^{2} ; \mu_{1}\right), \\
\left.b_{s+2}^{\times}\left(\nu ; 0 ; \mu_{1} ; 1 ; \vec{g}_{0}^{\vee}\right)\right|_{s \in \mathbb{N}} & =\left[\prod_{\ell=3}^{s+2} u_{\ell}^{\times}(\nu ; 0 ; 1)\right] b_{2}^{\times}\left(\nu ; 0 ; \mu_{1} ; 1 ; \vec{g}_{0}^{\vee}\right)+U\left(\nu^{2} ; \mu_{1}\right) \chi_{s ; 1}^{\wedge}(\nu ; 0 ; 1),
\end{aligned}
$$

DIFFERENTIAL EQUATIONS Vol. 51 No. $5 \quad 2015$ 
where $U\left(\nu^{2} ; \mu_{1}\right)=7 f_{3} \mathrm{P}_{3}\left(\mu_{1}\right)\left(\varkappa_{3}^{\times}(0 ; 1) \wp_{3}\left(\nu^{2} ; 0 ; 1\right)\right)^{-1},\left.\chi_{s ; s}^{\wedge}(\nu ; 0 ; 1)\right|_{s \in \mathbb{N}}=1$, and

$$
\chi_{s ; j}^{\wedge}(\nu ; 0 ; 1)=\prod_{r=j+3}^{s+2} u_{r}^{\times}(\nu ; 0 ; 1)
$$

for arbitrary $s \in \mathbb{N} \backslash\{1\}$ and $j \in\{1, \ldots, s-1\}$.

4. If $n^{*} \geq 4$, then

$$
\begin{aligned}
& b_{2}^{\times}\left(\nu ; 0 ; \mu_{1} ; 1 ; \vec{g}_{0}^{\vee}\right)=\left(\varkappa_{2}^{\times}(0 ; 1) \wp_{2}\left(\nu^{2} ; 0 ; 1\right)\right)^{-1} \sum_{j=0}^{n^{*}-2}(2 j+5) \Psi_{j}^{\wedge}(\nu ; 0 ; 1)\left(f_{j+2} \mathrm{P}_{j+2}\left(\mu_{1}\right)-\frac{2 \delta_{0 j}}{5}\right), \\
& \left.\sigma_{s}^{\times}\left(\nu ; 0 ; \mu_{1} ; 1\right)\right|_{s \in\left\{n^{*}-3, \ldots, 1\right\}} \\
& \quad=\left(\varkappa_{s+2}^{\times}(0 ; 1) \wp_{s+2}\left(\nu^{2} ; 0 ; 1\right)\right)^{-1}\left[(s+3) i \nu \sigma_{s+1}^{\wedge}\left(\nu ; 0 ; \mu_{1} ; 1\right)+(2 s+5) f_{s+2} \mathrm{P}_{s+2}\left(\mu_{1}\right)\right]
\end{aligned}
$$

and

$$
\begin{aligned}
& \left.\sigma_{s}^{\wedge}\left(\nu ; 0 ; \mu_{1} ; 1\right)\right|_{s \geq n^{*}-1}=0, \\
& \left.b_{s+2}^{\times}\left(\nu ; 0 ; \mu_{1} ; 1 ; \vec{g}_{0}^{\vee}\right)\right|_{s \in \mathbb{N}}=u_{s+2}^{\times}(\nu ; 0 ; 1) b_{s+1}^{\times}\left(\nu ; 0 ; \mu_{1} ; 1 ; \vec{g}_{0}^{\vee}\right)+\sigma_{s}^{\wedge}\left(\nu ; 0 ; \mu_{1} ; 1\right), \\
& \left.b_{s+2}^{\times}\left(\nu ; 0 ; \mu_{1} ; 1 ; \vec{g}_{0}^{\vee}\right)\right|_{s \in \mathbb{N}}=\left[\prod_{\ell=3}^{s+2} u_{\ell}^{\times}(\nu ; 0 ; 1)\right] b_{2}^{\times}\left(\nu ; 0 ; \mu_{1} ; 1 ; \vec{g}_{0}^{\vee}\right)+\sum_{j=1}^{\theta\left(s ; n^{*}-2\right)} \chi_{s ; j}^{\wedge}(\nu ; 0 ; 1) \sigma_{j}^{\wedge}\left(\nu ; 0 ; \mu_{1} ; 1\right), \\
& \left.b_{s}^{\times}\left(\nu ; 0 ; \mu_{1} ; 1 ; \vec{g}_{0}^{\vee}\right)\right|_{s \geq n^{*}+1}=\left[\prod_{\ell=n^{*}+1}^{s} u_{\ell}^{\times}(\nu ; 0 ; 1)\right] b_{n^{*}}^{\times}\left(\nu ; 0 ; \mu_{1} ; 1 ; \vec{g}_{0}^{\vee}\right) .
\end{aligned}
$$

Remark 6. Assume that $m=0, \omega_{0}=1, \nu \in G(0 ; 1)$, assumptions 2 and 3 of Theorem 12 are true, $\left\{b_{s}^{\times}\left(\nu ; 0 ; 1 ; \vec{g}_{0}^{\times}\right)\right\}_{s \in \mathbb{N}_{0}}=\left\{b_{s}^{\times}\left(\nu ; 0 ; \mu_{1} ; 1 ; \vec{g}_{0}^{\vee}\right)\right\}_{s \in \mathbb{N}_{0}}$ is the unique solution of system (4) in $\mathrm{W}_{\ell_{2 ; K_{2}(0)}}(G(0 ; 1))$, and the sequence $\left\{f_{j_{r}} \mathrm{P}_{j_{r}}\left(\mu_{1}\right)\right\}_{r \in \mathbb{N}}$ of all nonzero elements of the sequence $\vec{g}_{0}^{\vee}=\left\{f_{s} \mathrm{P}_{s}\left(\mu_{1}\right)\right\}_{s \in \mathbb{N}_{0}}$ is a countable set $\left(j_{r} \in \mathbb{N}_{0}\right.$ and $j_{r_{1}}<j_{r_{2}}$ for arbitrary $\left.r_{1}<r_{2}\right)$. Then the relation

$$
b_{s}^{\times}\left(\nu ; 0 ; \mu_{1} ; 1 ; \vec{g}_{0}^{\vee}\right)=\lim _{r \rightarrow+\infty} b_{s}^{\times}\left(\nu ; 0 ; \mu_{1} ; 1 ; \vec{g}_{0_{\left[0 ; j_{r}\right] ; \infty}^{\vee}}\right)
$$

holds for any finite number $s \in \mathbb{N}_{0}$, where $\vec{g}_{0_{\left[0 ; j_{r}\right] ; \infty}^{\vee}}^{\vee}=\left(f_{0} \mathrm{P}_{0}\left(\mu_{1}\right), \ldots, f_{j_{r}} \mathrm{P}_{j_{r}}\left(\mu_{1}\right), 0,0,0, \ldots\right)$. In this case, for any finite $r \in \mathbb{N}$ and for any $\nu \in G(0 ; 1)$, the number $b_{s}^{\times}\left(\nu ; 0 ; \mu_{1} ; 1 ; \vec{g}_{\left.0_{0 ;} ; j_{r}\right] ; \infty}^{\vee}\right)$ can be found with the use of the algorithm described in Theorem 13.

\section{ANALYTIC SOLUTIONS OF INHOMOGENEOUS SCALAR CHARACTERISTIC EQUATIONS OF THE THEORY OF RADIATION TRANSPORT}

The following assertion can be proved with the use of Theorems $2,3,5,12$, and 13 , Corollary 1 , and Remarks 2, 3, 5, and 6 .

Theorem 14. Let assumption 2 of Theorem 12 be satisfied, and let

$$
\nu \in G\left(|m| ; \omega_{0}\right)=\Upsilon \backslash \Re\left(|m| ; \omega_{0}\right)
$$

for some pair $\left(|m|, \omega_{0}\right) \in \mathbb{N}_{0} \times(0,1]$. Then, for arbitrary $\left(\mu, \mu_{1}\right) \in[-1,1] \times[-1,1]$, the resolvent $\Gamma_{|m|}\left(\nu ; \mu, \mu_{1} ; \omega_{0}\right)$ of the IE in the family of IE (3) corresponding to the pair $\left(|m|, \omega_{0}\right)$ can be represented in the form

$$
\begin{aligned}
& \Gamma_{|m|}\left(\nu ; \mu, \mu_{1} ; \omega_{0}\right)=\frac{\omega_{0}}{2(1-i \nu \mu)} \\
& \quad \times\left[\mathrm{P}_{|m|}\left(\mu, \mu_{1}\right)+\omega_{0} \sum_{s=0}^{+\infty}(2(s+|m|)+1) f_{s+|m|} \mathrm{P}_{s+|m|,|m|}^{\times}(\mu) b_{s}^{\times}\left(\nu ;|m| ; \mu_{1} ; \omega_{0} ; \vec{g}_{|m|}^{\vee}\right)\right],
\end{aligned}
$$


where $\left\{b_{s}^{\times}\left(\nu ;|m| ; \mu_{1} ; \omega_{0} ; \vec{g}_{|m|}^{\vee}\right)\right\}_{s \in \mathbb{N}_{0}}$ is the unique solution of the system in the family (4) corresponding to the index $m \in \mathbb{N}_{0}$ in $\mathrm{W}_{\ell_{2 ; \mathrm{K}_{2}(|m|)}}\left(G\left(|m| ; \omega_{0}\right)\right)$ for $\vec{g}_{m}^{\times}=\vec{g}_{|m|}^{\vee}=\left\{f_{s+|m|} \mathrm{P}_{s+|m|,|m|}^{\times}\left(\mu_{1}\right)\right\}_{s \in \mathbb{N}_{0}}$. In this case, the following assertions hold.

1. For each $\mu_{1} \in[-1,1]$, the series in $(28)$ is convergent pointwise and uniformly with respect to $\mu$ on the interval $[-1,1]$.

2. For arbitrary $\left(\mu, \mu_{1}\right) \in[-1,1] \times[-1,1]$, the sum of the series in (28) is an analytic function of $\nu \in G\left(|m| ; \omega_{0}\right)$.

3. If $\left(|m|, \omega_{0}\right) \in \mathbb{Q}$, then the sequence $\left\{b_{s}^{\times}\left(\nu ;|m| ; \mu_{1} ; \omega_{0} ; \vec{g}_{|m|}^{\vee}\right)\right\}_{s \in \mathbb{N}_{0}}$ can be found by the algorithm described in Theorem 12 and Remark 5.

4. If $m=0$ and $\omega_{0}=1$, then the sequence $\left\{b_{s}^{\times}\left(\nu ; 0 ; \mu_{1} ; 1 ; \vec{g}_{0}^{\vee}\right)\right\}_{s \in \mathbb{N}_{0}}$ is defined by the algorithm described in Theorem 13 and Remark 6.

The following assertion can be proved with the use of Theorems 1-5, Remark 2, the Fubini theorem, Theorem 2 in [12], the theorem on the addition of Legendre polynomials, Corollary 1, and the definition of resolvents of the IEs (1) and (3) and elements of the sequences $\vec{g}_{|m|}^{\vee}=\left\{g_{s}^{\vee}\left(|m| ; \mu_{1}\right)\right\}_{s \in \mathbb{N}_{0}}$ and $\left\{b_{s}^{\times}\left(\nu ;|m| ; \mu_{1} ; \omega_{0} ; \vec{g}_{|m|}^{\vee}\right)\right\}_{s \in \mathbb{N}_{0}}$.

Theorem 15. Let assumption 2 of Theorem 12 be satisfied, and let $\nu \in G\left(\omega_{0}\right)=\Upsilon \backslash \Re\left(\omega_{0}\right)$. Then the resolvent of the IE (1) can be represented in the analytic form

$$
\begin{aligned}
& \Gamma\left(\nu ; \vec{\Omega} ; \vec{\Omega}_{1} ; \omega_{0}\right) \\
& =\frac{\omega_{0}}{4 \pi(1-i \nu \mu)}\left[\mathrm{p}\left(\vec{\Omega} \cdot \vec{\Omega}_{1}\right)+\omega_{0} \sum_{s=0}^{+\infty} \sum_{m=0}^{s} w_{s m} \mathrm{P}_{s, m}^{\times}(\mu) b_{s-|m|}^{\times}\left(\nu ; m ; \mu_{1} ; \omega_{0} ; \vec{g}_{|m|}^{\vee}\right) \cos \left(m\left(\varphi-\varphi_{1}\right)\right)\right], \\
& \left(\vec{\Omega}, \vec{\Omega}_{1}, \omega_{0}\right) \in \Omega \times \Omega \times(0,1],
\end{aligned}
$$

where

$$
w_{s m}=(2 s+1) f_{s}\left(\delta_{0 m}+2\left(1-\delta_{0 s}\right)\left(1-\delta_{0 m}\right)\right) .
$$

In addition, for any $\vec{\Omega}_{1} \in \Omega$, the series in (29) is convergent pointwise and uniformly with respect to $\vec{\Omega}$ on $\Omega ;$ moreover, all elements of the sequences $\left\{b_{s}^{\times}\left(\nu ; m ; \mu_{1} ; \omega_{0} ; \vec{g}_{|m|}^{\vee}\right)\right\}_{(s ; m) \in \mathbb{N}_{0} \times\{0, \ldots, s\}}$ can be found with the use of the algorithms described in Theorems 12 and 13 and Remarks 5 and 6.

Remark 7. Let assumption 2 of Theorem 12 be satisfied for some pair

$$
\left(|m|, \omega_{0}\right) \in \mathbb{N}_{0} \times(0,1] \quad \nu=x+i y \in G\left(|m| ; \omega_{0}\right), \quad \vec{g}_{m}^{\times}=\vec{g}_{|m|}^{\vee}=\left\{f_{s+|m|} \mathrm{P}_{s+|m|,|m|}^{\times}\left(\mu_{1}\right)\right\}_{s \in \mathbb{N}_{0}},
$$

and let

$$
\vec{b}^{\times}\left(\nu ; m ; \omega_{0} ; \vec{g}_{m}^{\times}\right)=\vec{b}\left(\nu ;|m| ; \mu_{1} ; \omega_{0} ; \vec{g}_{|m|}^{\vee}\right)=\left\{b_{s}^{\times}\left(\nu ;|m| ; \mu_{1} ; \omega_{0} ; \vec{g}_{|m|}^{\vee}\right)\right\}_{s \in \mathbb{N}_{0}}
$$

be the unique solution of the corresponding system in the family (4) in the class

$$
\mathrm{W}_{\ell_{2 ; K_{2}\langle|m|)}}\left(G\left(|m| ; \omega_{0}\right)\right) \text {. }
$$

Then the following symmetry properties hold:

$$
\begin{aligned}
& \operatorname{Re}\left[b_{s}^{\times}\left(x+i y ;|m| ; \mu_{1} ; \omega_{0} ; \vec{g}_{|m|}^{\vee}\right)\right]=\operatorname{Re}\left[b_{s}^{\times}\left(-x+i y ;|m| ; \mu_{1} ; \omega_{0} ; \vec{g}_{|m|}^{\vee}\right)\right], \\
& \operatorname{Im}\left[b_{s}^{\times}\left(x+i y ;|m| ; \mu_{1} ; \omega_{0} ; \vec{g}_{|m|}^{\vee}\right)\right]=-\operatorname{Im}\left[b_{s}^{\times}\left(-x+i y ;|m| ; \mu_{1} ; \omega_{0} ; \vec{g}_{|m|}^{\vee}\right)\right], \quad s \in \mathbb{N}_{0} .
\end{aligned}
$$

The use of these symmetry properties permits dramatically reducing the amount of computations for finding numerical values of the resolvents $\Gamma_{|m|}\left(\nu ; \mu, \mu_{1} ; \omega_{0}\right)$ and $\Gamma\left(\nu ; \vec{\Omega}, \vec{\Omega}_{1} ; \omega_{0}\right)$ with the use of formulas (28) and (29). 


\section{APPENDIX}

Let us derive an analytic representation of the Green function

$$
\widetilde{G}_{\infty}\left(\tau, \vec{\Omega} ; \tau_{1}, \vec{\Omega}_{1} ; \omega_{0}\right)
$$

of the dimensionless scalar equation of radiation transport for the case of an infinite plane-parallel macroscopically homogeneous and locally isotropic disperse medium. This function is a solution of the following canonical BVP for the theory of the radiation transport [10]:

$$
\begin{aligned}
& \mu \frac{\partial \widetilde{G}_{\infty}\left(\tau, \vec{\Omega} ; \tau_{1}, \vec{\Omega}_{1} ; \omega_{0}\right)}{\partial \tau} \\
& =-\widetilde{G}_{\infty}\left(\tau, \vec{\Omega} ; \tau_{1}, \vec{\Omega}_{1} ; \omega_{0}\right)+\frac{\omega_{0}}{4 \pi} \int_{\Omega} \mathrm{p}\left(\vec{\Omega} \cdot \vec{\Omega}^{\prime}\right) \widetilde{G}_{\infty}\left(\tau, \vec{\Omega}^{\prime} ; \tau_{1}, \vec{\Omega}_{1} ; \omega_{0}\right) d \Omega^{\prime}+\delta\left(\tau-\tau_{1}\right) \delta\left(\mu-\mu_{1}\right) \delta\left(\varphi-\varphi_{1}\right), \\
& \left(\tau, \tau_{1}\right) \in \mathbb{R} \times \mathbb{R} \quad(\mathbb{R}=(-\infty,+\infty)), \quad \omega_{0} \in(0,1), \quad\left(\vec{\Omega}, \vec{\Omega}_{1}\right) \in \Omega \times \Omega, \\
& \lim _{\left|\tau-\tau_{1}\right| \rightarrow+\infty} \widetilde{G}_{\infty}\left(\tau, \vec{\Omega} ; \tau_{1}, \vec{\Omega}_{1} ; \omega_{0}\right)=0 .
\end{aligned}
$$

In system $(30), \delta(x)$ is the Dirac $\delta$ function. It was shown in [3] that

$$
\widetilde{G}_{\infty}\left(\tau, \vec{\Omega} ; \tau_{1}, \vec{\Omega}_{1} ; \omega_{0}\right)=\Theta\left(\tau-\tau_{1}, \mu\right) \delta\left(\mu-\mu_{1}\right) \delta\left(\varphi-\varphi_{1}\right)+\widetilde{G}_{\infty}^{1}\left(\tau, \vec{\Omega} ; \tau_{1}, \vec{\Omega}_{1} ; \omega_{0}\right),
$$

where

$$
\Theta(\tau, \mu)=\theta(\tau \mu)|\mu|^{-1} \exp (-|\tau / \mu|)
$$

$[\theta(\tau)=1$ for $\tau>0$ and $\theta(\tau)=0$ for $\tau<0]$, and the function $\widetilde{G}_{\infty}^{1}\left(\tau, \vec{\Omega} ; \tau_{1}, \vec{\Omega}_{1} ; \omega_{0}\right)$ is a solution of the BVP that coincides with the BVP (30) with the functions

$$
\widetilde{G}_{\infty}\left(\tau, \vec{\Omega} ; \tau_{1}, \vec{\Omega}_{1} ; \omega_{0}\right), \quad \delta\left(\tau-\tau_{1}\right) \delta\left(\mu-\mu_{1}\right) \delta\left(\varphi-\varphi_{1}\right)
$$

replaced by the functions $\widetilde{G}_{\infty}^{1}\left(\tau, \vec{\Omega} ; \tau_{1}, \vec{\Omega}_{1} ; \omega_{0}\right)$ and $\left(\omega_{0} /(4 \pi)\right) \Theta\left(\tau-\tau_{1}, \mu_{1}\right) \mathrm{P}\left(\vec{\Omega} \cdot \vec{\Omega}_{1}\right)$, respectively. Let the function $\mathrm{p}(\mu)$ satisfy the Hölder condition [13] on $[-1,1]$. Then, by using the Case method [8], one can show that if at least one of the inequalities $\mu \neq 0$ and $\mu_{1} \neq 0$ is satisfied, then $\widetilde{G}_{\infty}^{1}\left(\tau, \vec{\Omega} ; \tau_{1}, \vec{\Omega}_{1} ; \omega_{0}\right) \in L_{1}(-\infty,+\infty)$ as a function of the variable $\tau$. Under these assumptions, by taking into account the linearity of the BVP for the function $\widetilde{G}_{\infty}^{1}\left(\tau, \vec{\Omega} ; \tau_{1}, \vec{\Omega}_{1} ; \omega_{0}\right)$ and the IE for the resolvent $\Gamma\left(\nu ; \vec{\Omega}, \vec{\Omega}_{1} ; \omega_{0}\right)$ and by using the Fourier transform, we obtain the relation

$$
\overline{\widetilde{G}}_{\infty}^{1}\left(\nu ; \vec{\Omega} ; \tau_{1} ; \vec{\Omega}_{1} ; \omega_{0}\right)=\left(\sqrt{2 \pi}\left(1-i \nu \mu_{1}\right)\right)^{-1} \exp \left(i \nu \tau_{1}\right) \Gamma\left(\nu ; \vec{\Omega} ; \vec{\Omega}_{1} ; \omega_{0}\right),
$$

where $\nu$ is the parameter of the Fourier transform $[\nu \in(-\infty,+\infty)]$ and the function $\widetilde{G}_{\infty}^{1}\left(\nu, \vec{\Omega} ; \tau_{1}, \vec{\Omega}_{1} ; \omega_{0}\right)$ is the Fourier range of the function $\widetilde{G}_{\infty}^{1}\left(\tau, \vec{\Omega} ; \tau_{1}, \vec{\Omega}_{1} ; \omega_{0}\right)$. By using Theorem 4 and the IE (13), we show that the asymptotics $\left|\Gamma^{1}\left(\nu ; \vec{\Omega} ; \vec{\Omega}_{1} ; \omega_{0}\right)\right|=O\left(|\nu|^{-1} \ln |\nu|\right)$ holds for any $\omega_{0} \in(0,1)$ as $\nu \rightarrow \pm \infty$. By taking into account the relations in this section, Theorem 4 , and the theorem on the convolution with the use of the inverse Fourier transform, we obtain the desired analytic representation

$$
\begin{aligned}
& \widetilde{G}_{\infty}\left(\tau, \vec{\Omega} ; \tau_{1}, \vec{\Omega}_{1} ; \omega_{0}\right) \\
& =\Theta\left(\tau-\tau_{1}, \mu\right) \delta\left(\mu-\mu_{1}\right) \delta\left(\varphi-\varphi_{1}\right)+\frac{\omega_{0}}{4 \pi} \mathrm{p}\left(\vec{\Omega} \cdot \vec{\Omega}_{1}\right) \int_{-\infty}^{+\infty} \Theta(\tau-t, \mu) \Theta\left(t-\tau_{1}, \mu_{1}\right) d t \\
& \quad+\frac{\omega_{0}^{2}}{8 \pi^{2}} \int_{-\infty}^{+\infty} \frac{\exp \left(-i \nu\left(\tau-\tau_{1}\right)\right)}{(1-i \nu \mu)\left(1-i \nu \mu_{1}\right)}\left[\sum_{s=0}^{+\infty} \sum_{m=0}^{s} w_{s m} \mathrm{P}_{s, m}^{\times}(\mu) b_{s-|m|}^{\times}\left(\nu ; m ; \mu_{1} ; \omega_{0} ; \vec{g}_{m}^{\vee}\right) \cos \left(m\left(\varphi-\varphi_{1}\right)\right)\right] d \nu
\end{aligned}
$$


where $\mu_{1} \neq 0$. In this case, the second integral on the right-hand side in the representation (31) is convergent absolutely for arbitrary $\left(\tau, \tau_{1}, \vec{\Omega}, \vec{\Omega}_{1}, \omega_{0}\right) \in \mathbb{R} \times \mathbb{R} \times \Omega \times \Omega_{0} \times(0,1)$, where $\Omega_{0}$ is the unit sphere after the deletion of the unit circle defined by the conditions $\theta_{1}=(\pi / 2), \varphi \in[-\pi, \pi]$. To find the Green function for $\mu_{1}= \pm 0$, one can use its symmetry

$$
\widetilde{G}_{\infty}\left(\tau, \vec{\Omega} ; \tau_{1}, \vec{\Omega}_{1} ; \omega_{0}\right)=\widetilde{G}_{\infty}\left(\tau, \vec{\Omega}_{1} ; \tau_{1}, \vec{\Omega} ; \omega_{0}\right)
$$

The validity of relation (32) follows directly from the mutual principle [8, Chap. 2] and the invariance of the solution of the BVP (30) under orthogonal transformations of the Euclidean point space $\mathcal{E}_{3}$ which preserve the direction of the unit vector $\vec{e}_{1}$ or change it to the opposite direction. In the derivation of the Green function for $\mu_{1}= \pm 0$ on the basis of relations (31) and (32), one should assume that $\mu \neq 0$ and treat $\vec{g}_{|m|}^{\vee}$ as the sequence $\left\{f_{s+|m|} \mathrm{P}_{s+|m|,|m|}(\mu)\right\}_{s \in \mathbb{N}_{0}}$.

In conclusion, note that, by using the analytic representation (31) and the GIRRM [9, 10], one can efficiently solve BVP for a scalar RTE in the case of practically arbitrary phase functions and disperse media of various configurations.

\section{REFERENCES}

1. Rogovtsov, N.N., Constructive Theory of Scalar Characteristic Equations of the Theory of Radiation Transport: I. Basic Assertions of the Theory and Conditions for the Applicability of the Truncation Method, Differ. Uravn., 2015, vol. 51, no. 2, pp. 263-276.

2. Rogovtsov, N.N., On the Solution of the Characteristic Equation of the Theory of Radiation Transport in a Closed Form, Tr. mezhdunar. konf. "Kraevye zadachi, spetsial'nye funktsii i drobnoe ischislenie" (Proc. Int. Conf. "Boundary Value Problems, Special Functions, and Fractional Calculus"), Kilbas, A.A., Ed., Minsk, 1996, pp. 305-312.

3. Rogovtsov, N.N. and Borovik, F.N., The Characteristic Equation of Radiative Transfer Theory, in Light Scattering Reviews, Kokhanovsky, A.A., Ed., Chichester, 2009, vol. 4, pp. 347-429.

4. Rogovtsov, N.N., On Finding the Discrete Spectrum of Characteristic Equation of the Theory of the Radiation Transport on the Basis of the Study of the Convergence of the Sequence of Zeros of an Infinite System of Sturm Polynomials, Tr. mezhdunar. nauchno-tekhn. konf. "Nauka obrazovaniyu, proizvodstvu, ekonomike" (Proc. Int. Sci-Techn. Conf. "Science for Education, Industry, and Economics"), Khrustalev, B.M., Romanyuk, F.A., and Kalinichenko, A.S., Eds., Minsk, 2004, vol. 2, pp. 275-279.

5. Jones, W.B. and Thron, W.J., Continued Fractions. Analytic Theory and Applications, Reading: Addison-Wesley Publ., 1980.

6. Lorentzen, L. and Waadelend, H., Continued Fractions with Applications, Amsterdam, 1992.

7. Demidovich, B.P. and Maron, I.A., Osnovy vychislitel'noi matematiki (Foundations of Numerical Analysis), Moscow: Gosudarstv. Izdat. Fiz.-Mat. Lit., 1963.

8. Case, K.M. and Zweifel, P.F., Linear Transport Theory, Reading, MA, 1967.

9. Rogovtsov, N.N., Svoistva i printsipy invariantnosti. Prilozhenie $k$ resheniyu zadach matematicheskoi fiziki (Properties and Principles of Invariance. Application to Solution of Problems of Mathematical Physics), Minsk, 1999, part 1.

10. Rogovtsov, N.N., General Invariance Relations Reduction Method and Its Applications to Solutions of Radiative Transfer Problems for Turbid Media of Various Configurations, in Light Scattering Reviews, Kokhanovsky, A.A., Ed., Chichester, 2010, vol. 5, pp. 249-327.

11. Samarskii, A.A. and Nikolaev, E.S., Metody resheniya setochnykh uravnenii (Methods for the Solution of Difference Equations), Moscow: Nauka, 1978.

12. Maslennikov, M.A., The Milne Problem with Anisotropic Scattering, Tr. Mat. Inst. Steklova, 1968, vol. 47 , pp. 3-132.

13. Gakhov, F.D., Kraevye zadachi (Boundary Value Problems), Moscow: Nauka, 1977. 